\title{
Mitotic Analysis in Thirteen Varieties of Cajanus cajon (L.) Mill sp.
}

\author{
S. S. N. Sinha' and P. Kumar \\ Indian Lac Research Institute, Namkum, Ranchi-834010, India
}

Received October 29, 1977

The varieties of $C$. cajan appear to be distinct from each other on the basis of marked variation in their morphological characters, especially in colour and size of flowers, seed coats and their habitats which have led to the isolation of their 86 types (Shaw et al. 1935). These facts offer an opportunity for the cytologists to study the different varieties at cytological level. Moreover, scientific literature on $C$. cajan reveals that little attention has been paid to its cytological details (Krishnaswamy and Ayyanger 1935, Nathaini 1941 and Srivastava et al. 1973).

Therefore, an effort has been made to study the karyotypes of thirteen varieties of C. cajan (all of them different from those reported by Srivastava et al. (1973) in order to find out the differences in their finer details.

\section{Materials and methods}

The seeds of thirteen varieties of $C$. cajan (the varieties not yet catalogued were named after the place of their extensive cultivation) collected from various sources were germinated under laboratory conditions at room temperature $\left(22^{\circ} \mathrm{C} \pm\right.$ 2). All the cytological observations and camera lucida drawings were made from temporary acetocarmine (1 percent) squash preparations using paradichlorobenzene as pretreating agent.

The chromosome measuring $2.6 \mu$ and above were classified as A, 2.1 to $2.5 \mu$ as $\mathrm{B}, 1.6$ to $2.0 \mu$ as $\mathrm{C}$ and 1.0 to $1.5 \mu$ as $\mathrm{D}$ types. (The eye estimations were used wherever necessary). The arm ratio values reaching 1.0 were classified as Median (M), 1.1 to 1.9 as Submedian (SM) and 1.9 and above as Subterminal (ST) types. The $\mathrm{F}$ percent was calculated as short arm length/chromosome length $\times 100$. The $T F$ percent was calculated as total sum of short arm length/ total sum of chromosome length $\times 100$ (Huziwara 1962).

The cytological findings from an average of ten somatic metaphase plates were statistically analysed on the basis of $\mathrm{D}^{2}$-analysis, for which, following data were scored for individual chromosome in each variety i) the relative length of individual chromosome transformed into angular scale $=\mathrm{p}$ ard ii) the arm ratio transformed into logarithmic scale $=\mathrm{q}$. The chromosomes were recorded as $1,2,3,4,5,6,7,8,9,10$, and 11 to the values of their absolute length in descending

1 Professor-in-charge Cytogenetics and Radiation Genetics Laboratory, Deptt. of Botany, Ranchi University, Ranchi- 834008, India. 
order. For the convenience of grouping and calculations the varieties having SAT chromosomes were also included with other varieties for this study. Treating $\mathrm{p}$ as abscissa and $\mathrm{q}$ as ordinate each chromosome can be plotted as point $\mathrm{p}$ and $\mathrm{q}$, the graph will be straight line. The $\mathrm{p} / \mathrm{q}$ will show the gradient of the line $(\tan \theta)$, the length of the line being $=\left(\mathrm{p}^{2}+\mathrm{q}^{2}\right) \frac{1}{2}=\mathrm{Z}$ (say). The ratio $\tan \theta / \mathrm{Z}=\mathrm{K}$ (say) will give a good key to all quantitative characters of an individual chromosome. Let $\mathrm{Ki}^{\mathrm{x}}$ and $\mathrm{Ki}^{\mathrm{y}}$ be the $\mathrm{K}$ values in varieties $\mathrm{X}$ and $\mathrm{Y}$ respectively, then for eleven chromosomes which are independent variables, the degree of divergence between varieties $\mathrm{X}$ and $\mathrm{Y}$ will be $\mathrm{D}^{2}=\sum_{j}^{i}\left(\mathrm{Ki}^{\mathrm{x}}-\mathrm{Ki}^{\mathrm{y}}\right)^{2}$.

\section{Observations}

The observations on morphological details of various aspects of karyotypes of different varieties set out in Table 1 clearly reveal var. Ranchi having minimum total chromatin length $(35.4 \mu)$ and var. NP 41 the maximum $(51.2 \mu)$. Rest of the varieties occupied the intermediate positions. While considering the mean length of different pairs of chromosomes var. No. 148 has the highest mean length $(2.2 \mu)$ and var. Ranchi the lowest $(1.60 \mu)$. However, when range of length of different pairs of chromosomes are taken into account var. NP 41 is noted to have the shortest range (2.0-2.7 $\mu$ ) and var. PT 301 the longest (1.4-3.0 $\mu$ ). It is rather interesting to find out that var. Ranchi has smallest chromosome $(1.0 \mu)$ and PT 301 the largest $(3.0 \mu)$. As regards the TF percentages of thirteen varieties it can be seen that var. Motihari gets the top place (45.05) while var. PT 301 remains at the bottom (37.88). According to $\mathrm{F}$ percentage var. $\mathrm{C} 11$ is found to have the narrow range (41.98-47.68) and var. Assam possesses the broadest range (33.33-50.00).

When asymmetrical karyotypes are taken into account the varieties PT 301, $I L R I$ and $7 \mathrm{~S}$ having all the chromosome pairs of submedian and subterminal types, top the list and var. Ranchi with a minimum number of submedian and subterminal chromosome pairs occupies the bottom. Secondary constrictions are noted in only one pair of chromosome in varieties T1, No. 148 and NP 80.

On the basis of $\mathrm{D}^{2}$ value the varieties can be classified into seven distinct groups. Those included in these different groups are varieties PT 301, ILRI and $7 \mathrm{~S}$ in group $\mathrm{A}$, varieties $\mathrm{T} 1$ and $\mathrm{Cl1}$ in group $\mathrm{B}$, varieties NP 39 and NP 80 in group C, varieties NP 41, Motihari and BR 60 in group $E$ whereas the rest of the varieties are quite distant from each other and hence they are treated as independent units. The data have been set out on Fig. 4 and the values of $D^{2}$ have been given in Table 3. It was observed by treating $p$ as abscissa and $q$ as ordinate, that the points $\mathrm{p}$ and $\mathrm{q}$ formed a straight line,but because of thirteen varieties included in the present study, the graph becomes very much complicated and it is not possible to illustrate the graph in the text. 


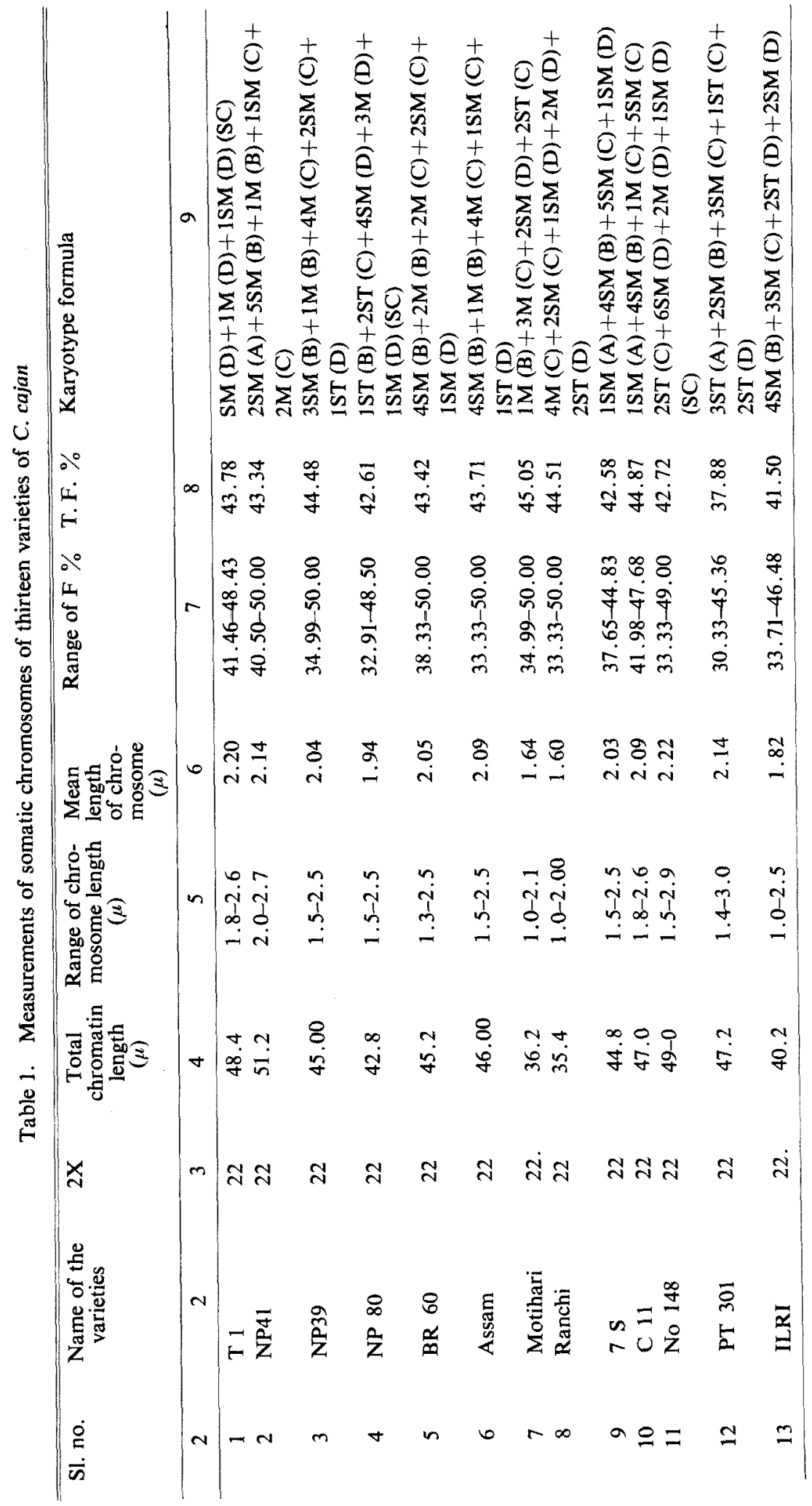


Table 2. Morphological characteristics

\begin{tabular}{|c|c|c|c|c|c|c|}
\hline Varieties & No. & Chromosome-1 & Chromosome-2 & Chromosome-3 & Chromosome-4 & Chromosome-5 \\
\hline 1 & 2 & 3 & 4 & 5 & 6 & 7 \\
\hline \multirow[t]{2}{*}{ T1 } & 1 & $\begin{array}{c}10.56 \pm 0.045 \\
(19.00)\end{array}$ & $\begin{array}{c}10.53 \pm 0.062 \\
(18.91)\end{array}$ & $\begin{array}{c}10.25 \pm 0.043 \\
(18.72)\end{array}$ & $\begin{array}{c}10.13 \pm 0.134 \\
(18.33)\end{array}$ & $\begin{array}{c}8.92 \pm 1.447 \\
(17.36))\end{array}$ \\
\hline & 2 & $\begin{array}{c}1.37 \pm 0.034 \\
(0.136)\end{array}$ & $\begin{array}{c}1.28 \pm 0.044 \\
(0.107)\end{array}$ & $\begin{array}{l}1.43 \pm 0.084 \\
(0.155)\end{array}$ & $\begin{array}{c}1.38 \pm 0.033 \\
(0.139)\end{array}$ & $\begin{array}{c}1.40 \pm 0.020 \\
(0.148)\end{array}$ \\
\hline \multirow[t]{2}{*}{ NP 41} & 1 & $\begin{array}{c}10.42 \pm 0.052 \\
(18.81)\end{array}$ & $\begin{array}{c}10.07 \pm 0.37 \\
(18.53)\end{array}$ & $\begin{array}{c}9.92 \pm 0.042 \\
(18.34)\end{array}$ & $\begin{array}{c}9.84 \pm 0.046 \\
(18.24)\end{array}$ & $\begin{array}{c}9.65 \pm 0.039 \\
(18.15)\end{array}$ \\
\hline & 2 & $\begin{array}{c}1.22 \pm 0.028 \\
(0.086)\end{array}$ & $\begin{array}{c}1.47 \pm 0.052 \\
(0.167)\end{array}$ & $\begin{array}{c}1.40 \pm 0.031 \\
(0.146)\end{array}$ & $\begin{array}{c}1.32 \pm 0.115 \\
(0.120)\end{array}$ & $\begin{array}{c}1.41 \pm 0.038 \\
(0.149)\end{array}$ \\
\hline \multirow[t]{2}{*}{ NP 39} & 1 & $\begin{array}{c}11.01 \pm 0.134 \\
(19.37)\end{array}$ & $\begin{array}{c}11.01 \pm 0.134 \\
(19.37)\end{array}$ & $\begin{array}{c}10.56 \pm 0.248 \\
(19.00)\end{array}$ & $\begin{array}{c}9.24 \pm 0.251 \\
(17.66)\end{array}$ & $\begin{array}{c}9.04 \pm 0.176 \\
(17.46)\end{array}$ \\
\hline & 2 & $\begin{array}{c}1.50 \pm 0.000 \\
(0.176)\end{array}$ & $\begin{array}{l}1.50 \quad 0.000 \\
(0.176)\end{array}$ & $\begin{array}{c}1.40 \pm 0.179 \\
(0.146)\end{array}$ & $\begin{array}{c}1.10 \pm 0.124 \\
(0.041)\end{array}$ & $\begin{array}{c}1.05 \pm 0.050 \\
(0.021)\end{array}$ \\
\hline \multirow[t]{2}{*}{ NP 80} & 1 & $\begin{array}{c}11.61+0.054 \\
(19.91)\end{array}$ & $\begin{array}{c}10.77 \pm 0.216 \\
(19.19)\end{array}$ & $\begin{array}{c}9.99 \pm 1.453 \\
(18.44)\end{array}$ & $\begin{array}{c}9.81 \pm 0.043 \\
(18.24)\end{array}$ & $\begin{array}{c}9.33 \pm 0.046 \\
(17.76)\end{array}$ \\
\hline & 2 & $\begin{array}{c}1.50 \pm 0.000 \\
(0.176)\end{array}$ & $\begin{array}{l}1.32 \pm 0.048 \\
(0.120)\end{array}$ & $\begin{array}{c}1.16 \pm 0.014 \\
(0.064)\end{array}$ & $\begin{array}{l}1.12 \pm 0.010 \\
(0.049)\end{array}$ & $\begin{array}{c}1.18 \pm 0.017 \\
(0.071)\end{array}$ \\
\hline \multirow[t]{2}{*}{ BR 60} & 1 & $\begin{array}{c}11.15 \pm 0.224 \\
(19.55)\end{array}$ & $\begin{array}{c}10.94+0.177 \\
(19.28)\end{array}$ & $\begin{array}{c}10.70 \pm 0.223 \\
(19.09)\end{array}$ & $\begin{array}{c}10.24 \pm 0.249 \\
(18.63)\end{array}$ & $\begin{array}{c}9.36 \pm 0.212 \\
(17.85)\end{array}$ \\
\hline & 2 & $\begin{array}{c}1.55 \pm 0.050 \\
(0.190)\end{array}$ & $\begin{array}{c}1.50 \pm 0.000 \\
(0.176)\end{array}$ & $\begin{array}{c}1.50 \pm 0.000 \\
(0.176)\end{array}$ & $\begin{array}{c}1.35 \pm 0.068 \\
(0.130)\end{array}$ & $\begin{array}{c}1.15 \pm 0.076 \\
(0.060)\end{array}$ \\
\hline \multirow[t]{2}{*}{ Assam } & 1 & $\begin{array}{c}10.78 \pm 0.110 \\
(19.19)\end{array}$ & $\begin{array}{c}10.78 \pm 0.110 \\
(19.19)\end{array}$ & $\begin{array}{c}10.78 \pm 0.110 \\
(19.19)\end{array}$ & $\begin{array}{c}10.33 \pm 0.233 \\
(18.72)\end{array}$ & $\begin{array}{l}9.25 \pm 0.272 \\
(17.76)\end{array}$ \\
\hline & 2 & $\begin{array}{c}1.50 \pm 0.000 \\
(0.176)\end{array}$ & $\begin{array}{c}1.50 \pm 0.00 \\
(0.176)\end{array}$ & $\begin{array}{c}1.50 \pm 0.000 \\
(0.176)\end{array}$ & $\begin{array}{c}1.50 \pm 0.066 \\
(0.176)\end{array}$ & $\begin{array}{c}1.15 \pm 0.076 \\
(0.060)\end{array}$ \\
\hline \multirow[t]{2}{*}{ Motihari } & i 1 & $\begin{array}{c}11.42 \pm 0.335 \\
(19.73)\end{array}$ & $\begin{array}{c}10.90 \pm 0.216 \\
(19.28)\end{array}$ & $\begin{array}{c}10.90 \pm 0.221 \\
(19.28)\end{array}$ & $\begin{array}{c}10.90 \pm 0.221 \\
(19.28)\end{array}$ & $\begin{array}{c}10.03 \pm 0.319 \\
(18.44)\end{array}$ \\
\hline & 2 & $\begin{array}{c}1.10 \pm 0.066 \\
(0.041)\end{array}$ & $\begin{array}{l}1.01 \pm 0.000 \\
(0.004)\end{array}$ & $\begin{array}{c}1.01 \pm 0.000 \\
(0.004)\end{array}$ & $\begin{array}{l}1.01 \pm 0.000 \\
(0.004)\end{array}$ & $\begin{array}{c}1.20 \pm 0.133 \\
(0.079)\end{array}$ \\
\hline \multirow[t]{2}{*}{ Ranchi } & 1 & $\begin{array}{c}11.21 \pm 0.267 \\
(19.55)\end{array}$ & $\begin{array}{c}11.21 \pm 0.267 \\
(19.55)\end{array}$ & $\begin{array}{c}11.11 \pm 0.150 \\
(19.46)\end{array}$ & $\begin{array}{c}11.11 \pm 0.150 \\
(19.46)\end{array}$ & $\begin{array}{c}10.05 \pm 0.378 \\
(18.53)\end{array}$ \\
\hline & 2 & $\begin{array}{l}1.01 \pm 0.000 \\
(0.004)\end{array}$ & $\begin{array}{l}1.01 \pm 0.000 \\
(0.004)\end{array}$ & $\begin{array}{c}1.01 \pm 0.000 \\
(0.004)\end{array}$ & $\begin{array}{l}1.01 \pm 0.000 \\
(0.004)\end{array}$ & $\begin{array}{c}1.40 \pm 0.160 \\
(0.146)\end{array}$ \\
\hline \multirow[t]{2}{*}{$7 \mathrm{~S}$} & 1 & $\begin{array}{c}12.13 \pm 0.135 \\
(20.36)\end{array}$ & $\begin{array}{c}10.48 \pm 0.062 \\
(18.91)\end{array}$ & $\begin{array}{c}10.15 \pm 0.122 \\
(18.53)\end{array}$ & $\begin{array}{l}9.89 \pm 0.124 \\
(18.34)\end{array}$ & $\begin{array}{c}9.36 \pm 0.096 \\
(17.85)\end{array}$ \\
\hline & 2 & $\begin{array}{l}1.25 \pm 0.004 \\
(0.096)\end{array}$ & $\begin{array}{l}1.24 \neq 0.028 \\
(0.093)\end{array}$ & $\begin{array}{l}1.23 \pm 0.028 \\
(0.089)\end{array}$ & $\begin{array}{l}1.31 \pm 0.036 \\
(0.117)\end{array}$ & $\begin{array}{l}1.28 \pm 0.014 \\
(0.107)\end{array}$ \\
\hline \multirow[t]{2}{*}{$\mathrm{Cll}$} & 1 & $\begin{array}{c}10.83 \pm 0.079 \\
(19.19)\end{array}$ & $\begin{array}{c}10.68 \pm 0.067 \\
(18.15)\end{array}$ & $\begin{array}{c}10.44 \pm 0.080 \\
\quad(18.81)\end{array}$ & $\begin{array}{c}9.88 \pm 0.198 \\
(18.34\end{array}$ & $\begin{array}{l}8.38 \pm 0.227 \\
(16.85)\end{array}$ \\
\hline & 2 & $\begin{array}{c}1.36 \pm 0.008 \\
(0.133)\end{array}$ & $\begin{array}{c}1.35 \pm 0.038 \\
(0.130)\end{array}$ & $\begin{array}{c}1.38 \pm 0.114 \\
(0.139)\end{array}$ & $\begin{array}{c}1.32 \pm 0.054 \\
(0.120)\end{array}$ & $\begin{array}{l}1.18 \pm 0.088 \\
(0.071)\end{array}$ \\
\hline \multirow[t]{2}{*}{ No 148} & 1 & $\begin{array}{c}12.02 \pm 1.265 \\
\quad(20.27)\end{array}$ & $\begin{array}{c}10.70 \pm 0.107 \\
(19.09)\end{array}$ & $\begin{array}{c}10.13 \pm 0.137 \\
(18.53)\end{array}$ & $\begin{array}{c}10.13 \pm 0.137 \\
(18.53)\end{array}$ & $\begin{array}{c}9.97 \pm 0.017 \\
(18.44)\end{array}$ \\
\hline & 2 & $\begin{array}{c}1.93 \pm 0.064 \\
(0.285)\end{array}$ & $\begin{array}{c}1.42 \pm 0.200 \\
(0.152)\end{array}$ & $\begin{array}{c}1.48 \pm 0.014 \\
(0.170)\end{array}$ & $\begin{array}{c}1.39 \pm 0.034 \\
(0.143)\end{array}$ & $\begin{array}{c}1.29 \pm 0.144 \\
(0.110)\end{array}$ \\
\hline \multirow[t]{2}{*}{ PT 301} & 1 & $\begin{array}{c}12.59 \pm 0.050 \\
(20.79)\end{array}$ & $\begin{array}{c}12.59 \pm 0.056 \\
(20.79)\end{array}$ & $\begin{array}{c}10.91 \pm 0.271 \\
(19.28)\end{array}$ & $\begin{array}{c}10.37 \pm 0.100 \\
(18.81)\end{array}$ & $\begin{array}{c}9.06 \pm 0.098 \\
(17.56)\end{array}$ \\
\hline & 2 & $\begin{array}{l}2.00 \pm 0.000 \\
(0.301)\end{array}$ & $\begin{array}{l}2.33+0.000 \\
(0.367)\end{array}$ & $\begin{array}{c}1.66 \pm 0.011 \\
(0.220)\end{array}$ & $\begin{array}{c}1.47 \pm 0.030 \\
(0.167)\end{array}$ & $\begin{array}{l}1.20 \pm 0.000 \\
(0.079)\end{array}$ \\
\hline \multirow[t]{2}{*}{ ILRI } & 1 & $\begin{array}{c}12.03 \pm 0.187 \\
(20.27)\end{array}$ & $\begin{array}{c}11.81 \pm 0.191 \\
(20.09)\end{array}$ & $\begin{array}{c}11.34 \pm 0.180 \\
(19.64)\end{array}$ & $\begin{array}{c}10.81 \pm 0.146 \\
(19.19)\end{array}$ & $\begin{array}{l}9.84 \pm 0.360 \\
\quad(18.24)\end{array}$ \\
\hline & 2 & $\begin{array}{c}1.45 \pm 0.022 \\
(0.161)\end{array}$ & $\begin{array}{l}1.41 \pm 0.022 \\
(0.149)\end{array}$ & $\begin{array}{l}1.31 \pm 0.026 \\
(0.117)\end{array}$ & $\begin{array}{l}1.21 \pm 0.040 \\
(0.082)\end{array}$ & $\begin{array}{c}1.18 \pm 0.096 \\
(0.071)\end{array}$ \\
\hline
\end{tabular}

$1=$ Relative length $\pm \mathrm{S}$. $\mathrm{E}$.

$(-)=$ Angular transformation value 
of karyotypes of Cajanus cajan

Chromosome-6 Chromosome-7 Chromosome-8 Chromosome-9 Chromosome-10 Chromosome-11

\begin{tabular}{|c|c|c|c|c|c|}
\hline 8 & 9 & 10 & 11 & 12 & 13 \\
\hline $\begin{array}{c}8.81 \pm 0.233 \\
(17.36)\end{array}$ & $\begin{array}{c}8.16 \pm 0.033 \\
(19.64)\end{array}$ & $\begin{array}{l}8.16 \pm 0.033 \\
(16.64)\end{array}$ & $\begin{array}{l}8.12 \pm 0.044 \\
(16.54)\end{array}$ & $\begin{array}{l}7.79+0.061 \\
(16.22)\end{array}$ & $\begin{array}{l}7.42 \pm 0.060 \\
(15.79)\end{array}$ \\
\hline $\begin{array}{c}1.16 \pm 0.064 \\
(0.064)\end{array}$ & $\begin{array}{c}1.06 \pm 0.057 \\
(0.025)\end{array}$ & $\begin{array}{l}1.23 \pm 0.051 \\
(0.089)\end{array}$ & $\begin{array}{c}1.41 \pm 0.037 \\
(0.149)\end{array}$ & $\begin{array}{c}1.26 \pm 0.033 \\
(0.100)\end{array}$ & $\begin{array}{c}1.33 \pm 0.054 \\
(0.123)\end{array}$ \\
\hline $\begin{array}{c}9.41 \pm 0.050 \\
(17.85)\end{array}$ & $\begin{array}{c}9.07 \pm 0.058 \\
(17.56)\end{array}$ & $\begin{array}{l}8.26 \pm 0.0461 \\
(16.74)\end{array}$ & $\begin{array}{l}7.72 \pm 0.037 \\
(16.11)\end{array}$ & $\begin{array}{l}7.72 \pm 0.037 \\
(16.11)\end{array}$ & $\begin{array}{c}7.62 \pm 0.401 \\
(16.00)\end{array}$ \\
\hline $\begin{array}{c}1.37 \pm 0.042 \\
(0.136)\end{array}$ & $\begin{array}{l}1.35 \pm 0.026 \\
(0.130)\end{array}$ & $\begin{array}{c}1.14 \pm 0.041 \\
(0.056)\end{array}$ & $\begin{array}{l}1.01 \pm 0.000 \\
(0.004)\end{array}$ & $\begin{array}{l}1.22 \pm 0.028 \\
(0.086)\end{array}$ & $\begin{array}{l}1.47 \pm 0.037 \\
(0.167)\end{array}$ \\
\hline $\begin{array}{l}8.83 \pm 0.106 \\
(17.26)\end{array}$ & $\begin{array}{l}8.75 \pm 0.409 \\
(17.26)\end{array}$ & $\begin{array}{l}8.51 \pm 0.428 \\
(16.95)\end{array}$ & $\begin{array}{l}8.14 \pm 0.272 \\
\quad(17.50)\end{array}$ & $\begin{array}{c}7.90 \pm 0.303 \\
(16.32)\end{array}$ & $\begin{array}{l}6.82 \pm 0.202 \\
\quad(15.12)\end{array}$ \\
\hline $\begin{array}{l}1.01 \pm 0.000 \\
(0.021)\end{array}$ & $\begin{array}{l}1.01 \pm 0-000 \\
(0.021)\end{array}$ & $\begin{array}{l}1.01 \neq 0.145 \\
(0.021)\end{array}$ & $\begin{array}{c}1.30 \pm 0.152 \\
(0.113)\end{array}$ & $\begin{array}{l}1.40 \pm 0.166 \\
(0.146)\end{array}$ & $\begin{array}{l}1.90 \div 0.100 \\
(0.278)\end{array}$ \\
\hline $\begin{array}{l}9.24 \pm 0.078 \\
(17.66)\end{array}$ & $\begin{array}{l}9.24 \pm 0.078 \\
(17.26)\end{array}$ & $\begin{array}{c}8.49 \pm 0.237 \\
(17.95)\end{array}$ & $\begin{array}{c}7.57 \pm 0.100 \\
(16.00)\end{array}$ & $\begin{array}{c}7.02 \pm 0.051 \\
(15.84)\end{array}$ & $\begin{array}{l}6.93 \pm 0.047 \\
(15.23)\end{array}$ \\
\hline $\begin{array}{l}1.08 \pm 0.030 \\
(0.033)\end{array}$ & $\begin{array}{l}1.04 \pm 0.024 \\
(0.071)\end{array}$ & $\begin{array}{c}1.48 \pm 0.222 \\
(0.167)\end{array}$ & $\begin{array}{l}2.18+0.181 \\
\quad(0.338)\end{array}$ & $\begin{array}{l}2.04 \pm 0.026 \\
(0.309)\end{array}$ & $\begin{array}{l}2.01 \pm 0.000 \\
(0.303)\end{array}$ \\
\hline $\begin{array}{c}9.15+0.200 \\
(17.66)\end{array}$ & $\begin{array}{c}8.95 \pm 0.186 \\
(17.46)\end{array}$ & $\begin{array}{l}8.75 \pm 0.140 \\
(17.26)\end{array}$ & $\begin{array}{l}7.83 \pm 0.281 \\
(16.22)\end{array}$ & $\begin{array}{c}7.38 \pm 0.269 \\
(15.79)\end{array}$ & $\begin{array}{l}5.67 \pm 0.340 \\
(13.81)\end{array}$ \\
\hline $\begin{array}{c}1.10 \pm 0.066 \\
(0.041)\end{array}$ & $\begin{array}{l}1.01 \pm 0.117 \\
(0.004)\end{array}$ & $\begin{array}{l}1.01 \pm 0.000 \\
(0.004)\end{array}$ & $\begin{array}{l}1.40 \pm 0.161 \\
(0.146)\end{array}$ & $\begin{array}{l}1.70 \pm 0.150 \\
(0.230)\end{array}$ & $\begin{array}{l}1.50 \pm 0.166 \\
(0.176)\end{array}$ \\
\hline $\begin{array}{l}8.62 \pm 0.087 \\
(17.05)\end{array}$ & $\begin{array}{c}8.62 \pm 0.087 \\
(17.05)\end{array}$ & $\begin{array}{l}8.39 \pm 0.161 \\
(16.85)\end{array}$ & $\begin{array}{l}8.39 \pm 0.048 \\
(16.85)\end{array}$ & $\begin{array}{c}7.52 \pm 0.321 \\
(15.89)\end{array}$ & $\begin{array}{c}6.45 \pm 0.122 \\
(14.77)\end{array}$ \\
\hline $\begin{array}{l}1.05 \pm 0.050 \\
(0.021)\end{array}$ & $\begin{array}{l}1.01 \pm 0.000 \\
(0.004)\end{array}$ & $\begin{array}{l}1.10 \pm 0.100 \\
(0.041)\end{array}$ & $\begin{array}{c}1.10 \neq 0.100 \\
(0.041)\end{array}$ & $\begin{array}{c}1.40 \pm 0.160 \\
(0.146)\end{array}$ & $\begin{array}{l}2.00 \pm 0.000 \\
(0.301)\end{array}$ \\
\hline $\begin{array}{c}9.46 \pm 0.285 \\
(17.95)\end{array}$ & $\begin{array}{l}8.93 \pm 0.270 \\
(17.36)\end{array}$ & $\begin{array}{l}8.42 \pm 0.199 \\
(16.85)\end{array}$ & $\begin{array}{c}7.30 \pm 0.341 \\
(15.68)\end{array}$ & $\begin{array}{l}6.25 \pm 0.577 \\
(14.42)\end{array}$ & $\begin{array}{l}5.69 \pm 0.734 \\
(13.81)\end{array}$ \\
\hline $\begin{array}{c}1.50 \pm 0.166 \\
(0.176)\end{array}$ & $\begin{array}{l}1.70 \pm 0.152 \\
(0.230)\end{array}$ & $\begin{array}{c}1.90 \pm 0.179 \\
(0.278)\end{array}$ & $\begin{array}{l}1.90 \pm 0.100 \\
(0.278)\end{array}$ & $\begin{array}{c}1.40 \pm 0.066 \\
(0.146)\end{array}$ & $\begin{array}{c}1.30 \pm 0.152 \\
(0.113)\end{array}$ \\
\hline $\begin{array}{c}9.49+0.354 \\
(17.95)\end{array}$ & $\begin{array}{c}8.61 \pm 0.163 \\
(17.05)\end{array}$ & $\begin{array}{c}8.61 \pm 0.163 \\
(17.05)\end{array}$ & $\begin{array}{c}7.53 \pm 0.379 \\
(15.89)\end{array}$ & $\begin{array}{l}5.86 \pm 0.230 \\
\quad(14.06)\end{array}$ & $\begin{array}{c}5.60 \pm 0.163 \\
(13.69)\end{array}$ \\
\hline $\begin{array}{l}1.60 \pm 0.160 \\
(0.204)\end{array}$ & $\begin{array}{l}2.00 \pm 0.000 \\
(0.301)\end{array}$ & $\begin{array}{l}2.00 \pm 0.000 \\
(0.301)\end{array}$ & $\begin{array}{l}1.70 \pm 0.152 \\
\quad(0.230)\end{array}$ & $\begin{array}{l}1.10 \pm 0.100 \\
(0.041)\end{array}$ & $\begin{array}{l}1.01 \pm 0.000 \\
(0.004)\end{array}$ \\
\hline $\begin{array}{c}9.05 \pm 0.081 \\
(17.56)\end{array}$ & $\begin{array}{c}9.01 \neq 0.098 \\
(17.46)\end{array}$ & $\begin{array}{l}8.83 \pm 0.131 \\
\quad(17.26)\end{array}$ & $\begin{array}{c}8.61 \pm 0.070 \\
(17.05)\end{array}$ & $\begin{array}{c}7.09 \pm 0.356 \\
(15.45)\end{array}$ & $\begin{array}{l}5.10 \pm 0.160 \\
(13.05-\end{array}$ \\
\hline $\begin{array}{l}1.22 \pm 0.009 \\
(0.086)\end{array}$ & $\begin{array}{l}1.32 \pm 0.046 \\
(0.120)\end{array}$ & $\begin{array}{l}1.44 \pm 0.026 \\
(0.158)\end{array}$ & $\begin{array}{l}1.64 \pm 0.031 \\
(0.214)\end{array}$ & $\begin{array}{l}1.65 \pm 0.014 \\
(0.217)\end{array}$ & $\begin{array}{l}1.55 \pm 0.014 \\
(0.190)\end{array}$ \\
\hline $\begin{array}{l}8.30 \pm 0.185 \\
(16.74)\end{array}$ & $\begin{array}{c}9.12 \pm 0.046 \\
(17.56)\end{array}$ & $\begin{array}{c}8.59 \pm 0.050 \\
(17.05)\end{array}$ & $\begin{array}{l}8.22 \pm 0.040 \\
(16.64)\end{array}$ & $\begin{array}{l}7.92 \pm 0.064 \\
(16.32)\end{array}$ & $\begin{array}{l}7.55 \pm 0.061 \\
(16.00)\end{array}$ \\
\hline $\begin{array}{l}1.06 \pm 0.040 \\
(0.025)\end{array}$ & $\begin{array}{l}1.17 \pm 0.040 \\
(0.068)\end{array}$ & $\begin{array}{c}1.25 \pm 0.063 \\
(0.096)\end{array}$ & $\begin{array}{l}1.38 \pm 0.059 \\
(0.139)\end{array}$ & $\begin{array}{l}1.21 \pm 0.033 \\
(0.082)\end{array}$ & $\begin{array}{l}1.20 \pm 0.068 \\
(0.079)\end{array}$ \\
\hline $\begin{array}{c}8.83 \pm 0.202 \\
(17.26)\end{array}$ & $\begin{array}{l}8.30 \pm 0.065 \\
(16.74)\end{array}$ & $\begin{array}{l}8.14 \pm 0.036 \\
(16.54)\end{array}$ & $\begin{array}{c}8.10 \pm 0.055 \\
(16.54)\end{array}$ & $\begin{array}{l}7.69 \pm 0.244 \\
(16.11)\end{array}$ & $\begin{array}{c}6.08 \pm 0.041 \\
(14.30)\end{array}$ \\
\hline $\begin{array}{l}1.15 \pm 0.024 \\
(0.060)\end{array}$ & $\begin{array}{l}1.16 \pm 0.017 \\
(0.064)\end{array}$ & $\begin{array}{l}1.16 \pm 0.028 \\
(0.064)\end{array}$ & $\begin{array}{l}1.02 \pm 0.020 \\
(0.004)\end{array}$ & $\begin{array}{c}1.20 \pm 0.133 \\
(0.079)\end{array}$ & $\begin{array}{l}2.00 \pm 0.000 \\
(0.301)\end{array}$ \\
\hline $\begin{array}{l}8.40 \pm 0.043 \\
(16.85)\end{array}$ & $\begin{array}{l}8.39 \pm 0.040 \\
(16.85)\end{array}$ & $\begin{array}{l}8.39+0.040 \\
(16.85)\end{array}$ & $\begin{array}{c}7.16 \pm 0.307 \\
(15.45)\end{array}$ & $\begin{array}{c}6.17 \pm 0.060 \\
(14.42)\end{array}$ & $\begin{array}{c}5.93 \pm 0.076 \\
(14.06)\end{array}$ \\
\hline $\begin{array}{l}1.22 \pm 0.006 \\
(\mathbf{0 . 0 8 8 )}\end{array}$ & $\begin{array}{l}1.30 \pm 0.042 \\
(0.113)\end{array}$ & $\begin{array}{c}1.47 \neq 0.035 \\
(0.167)\end{array}$ & $\begin{array}{l}1.80 \pm 0.024 \\
(0.255)\end{array}$ & $\begin{array}{l}1.94 \pm 0.010 \\
(0.287)\end{array}$ & $\begin{array}{l}1.80 \pm 0.000 \\
(0.225)\end{array}$ \\
\hline $\begin{array}{l}9.75 \pm 0.247 \\
\quad(18.05)\end{array}$ & $\begin{array}{c}8.44 \pm 0.330 \\
(16.85)\end{array}$ & $\begin{array}{c}7.62 \pm 0.273 \\
(16.00)\end{array}$ & $\begin{array}{l}7.19 \pm 0.219 \\
\quad(15.56)\end{array}$ & $\begin{array}{c}6.17 \pm 0.280 \\
(14.42)\end{array}$ & $\begin{array}{l}4.95 \pm 0.060 \\
(12.92)\end{array}$ \\
\hline $\begin{array}{l}1.22 \pm 0.111 \\
(0.086)\end{array}$ & $\begin{array}{l}1.54 \pm 0.170 \\
(0.187)\end{array}$ & $\begin{array}{l}1.92 \pm 0.114 \\
(0.283)\end{array}$ & $\begin{array}{l}2.02 \pm 0.135 \\
(0.305)\end{array}$ & $\begin{array}{l}1.50 \pm 0.115 \\
(0.176)\end{array}$ & $\begin{array}{c}1.20 \pm 0.143 \\
(0.079)\end{array}$ \\
\hline
\end{tabular}




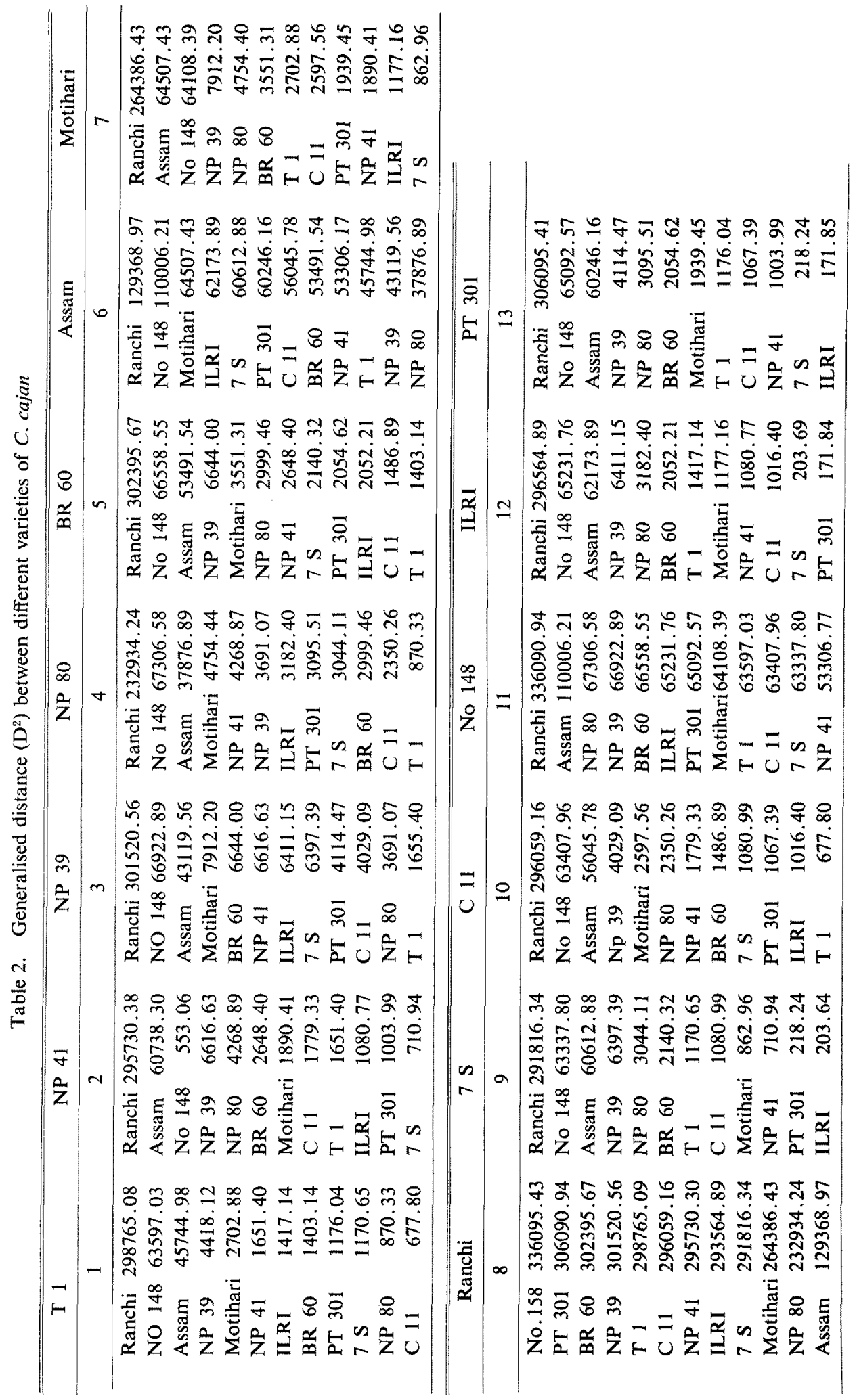



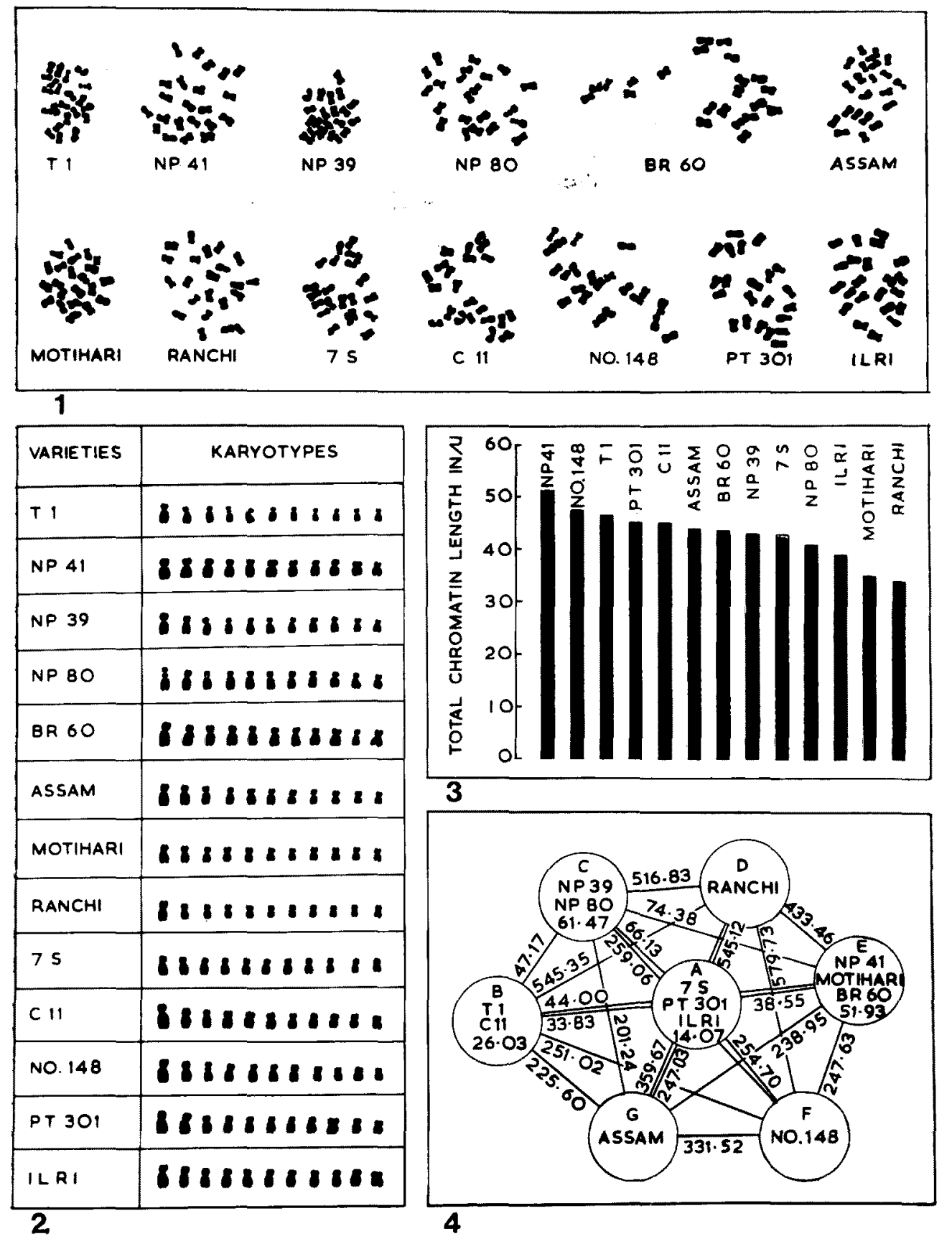

Figs. 1-4. 1, somatic metaphase chromosomes of thirteen varieties of Cajanus cajan, $\times 1200$. Idiograms showing haploid karyotypes of thirteen varieties of Cajanus cajan. 3, histogram showing total chromatin length of thirteen varieties of Cajanus cajan. 4, graphic representation of $\mathrm{D}^{2}$ (distance) values between thirteen varieties of Cajanus cajan. 


\section{Discussion}

The present investigation incorporates the karyotypic analysis in thirteen varieties of $C$. cajan, all of which have $2 n=22$. All the varieties under study are diploids and hence the species may be considered as homogeneous monobasic one. Such a similarity in chromosome number of these varieties of $C$. cajan indicates towards their evolution from a common ancestor which has formed an uniform congregation. However the differences in their respective karyotypes are also quite substantial and may explain the variation within the species. The differences in the total chromatin length can be regarded as one of the most important factors in their evolutionary history. According to Babcock and Cameron (1934) decreases in total chromatin length is one of the factors responsible for evolution and hence var. Ranchi and Motihari having minimum lengths may be considered as most advanced and var. NP 41 with maximum chromatin length as most primitive. Such a reduction in chromatin length appears to occur due to the erosion of the chromatid segments during the process of evolution.

When the karyotypic asymmetry is taken into account the asymmetrical karyotypes are supposed to be more advanced than symmetrical ones (Stebbins 1950 ) and therefore, the varieties having maximum number of submedian chromosomes (asymmetrical) are the more advanced than those having minimum number of submedian chromosomes (Table 1). On the basis of TF percent also var. PT 301 tends to be the most advanced having the smallest TF percent and var. Motihari possessing the largest TF percent, may be considered as the most primitive one. The secondary constrictions have been recorded only in the varieties T1, No. 148 and NP 80 (Fig. 2). The absence of secondary constrictions in all other varieties may be attributed to the fact that accidental hybridization and translocation might have occurred during the course of evolution resulting in the elimination of SAT chromosomes as also reported by Delauney (1926), Sato (1937) and Sinha and Acharia (1972). In some of the varieties like NP 39, NP 80, Assam, Motihari, Ranchi, No. 148, PT 301 and ILRI chromosomes with subterminal centromeres are present. Such a situation may arise due to deletion and deficiency in one arm of the chromosomes causing the shift in centromeric positions. This shifting of the centrmomeric position may not be in a similar fashion in all the chromosomes of the same variety and thus lead to asymmetrical condition of the karyotypes. This karyotypic evolution brought about by repatterning of chromosomes might be considered as one of the prime factors for evolution within the same species and thus the formation of different varieties with the same chromosome numbers.

According to $\mathrm{D}^{2}$ analysis cluster $\mathrm{A}$-includes var. PT 301, ILRI and 7S; Cluster B-var. T1, C11; Cluster C-var NP 39, NP 80 and Cluster E-var. NP 41, Motihari and BR 60 on the basis of lesser distances amongst each other. Rest of the varieties have formed their own clusters individually namely $D, F$ and $G$ including var. Ranchi, No. 148 and Assam respectively since they maintain a respectable distance amongst themselves and also from other clusters. The average minimum distance has been observed in cluster $A$ (14.07) and the maximum distance is noted in cluster C (61.47) whereas clusters B and E record distances 
as 26.03 and 51.93 respectively. While considering the intercluster distances we find that the maximum distance (579.73) exists in between clusters $D$ and $F$ and minimum (33.83) in between A and B (Fig. 4). It is also interesting to note that the varieties included in the same cluster are appreciably close to each other in other cytological characteristics too, however, their cytological dissimilarities tend to increase with the increase of generalised distance amongst them (Table 3). This analysis may be helpful in bringing the different varieties closer by putting them in the same cluster and thereby minimising the number of varieties. From the above discussion it becomes apparent that the grouping pattern according to $\mathrm{D}^{2}$ values gives a better insight into the finer details of cytological characteristics of the different varieties in the same species.

However, generally it is observed that all the evolutionary tendencies might not go hand in hand in the phylogenetic evolution of a taxon due to their different adaptive values. As such, one of the characters might put the variety at highest level of advancement while the others may be still in the primitive stage. It can be visualised in case of variety Ranchi in which, when the total chromatin length is considered it makes it highly advanced while on the basis of number of submedian chromosomes and asymmetrical karyotypes it can be classified as most primitive.

Thus on the basis of foregoing discussion it can easily be concluded that the karyotype evolution within the varieties might have been through quantitative or qualitative or both types of changes in the chromosome complement of the varieties. The formation of different varieties in $C$. cajan on the basis of cytological differences has also been reported by Srivastava et al. (1973) and the above discussion in the light of detailed investigations have further strengthened the view that the varietal differentiation appears to be accompanied with cytological variations.

\section{Summary}

The somatic chromosomes studied in the root tips of thirteen varieties of $C$. cajan revealed that all the varieties had the same chromosome number $(2 n=22)$ but there is much variation in their chromosome morphology. The total chromatin length was maximum $(51.2 \mu)$ in variety NP 41 and its minimum value was noted in variety Ranchi. When the individual chromosomes were taken into account the shortest $(1.0 \mu)$ and the largest $(3.0 \mu)$ chromosomes were recorded in var. Ranchi and var. PT 301 respectively. The TF percent was maximum in variety Mothihari (45.05) and minimum in variety PT 301. The SAT chromosomes were noted in varieties $T 1$, No 148 and NP 80 . On the basis of $D^{2}$ values the varieties were classified into seven distinct groups. (A-varieties PT 301; ILRI and 7S; B-T1 and C11; C-NP 39 and NP 80; D-Ranchi; E-NP 41, Motihari and BR $60 ; \mathrm{F}-\mathrm{No} 148$ and $\mathrm{G}-$ Assam). This indicates that the variety formation in $C$. cajan had taken place mainly due to the change in the chromosome morphology and nature of genes. 


\section{Acknowledgements}

This study is a part of the thesis submitted to the Ranchi University for the award of Ph. D. degree by the junior author.

The authors are extremely grateful to the Director, Indian Lac Research Institute, Namum, Ranchi and Dr. J. P. Sinha, Professor and Head of Botany Deptt., University of Ranchi, Ranchi for kindly according their permission to work and providing necessary facilities. Our thanks are also due to Sarvasree P. Das, R. L. Singh and J. N. Dwivedi for help in art and photographic work and R. C. Acharia and B. N. Sah for help in statistical analysis.

\section{References}

Babcock, E. B. and Cameron, D. R. 1934. Chromosome and phylogeny in Crepis II. The relationship of 108 species. Univ. Calif. Publ. Agr. Sci. 6: 287-324.

Delauney, L. 1926. Phylogenetische Chromosomenverkurzung. Zeitschr. Zellf. u. Mikr. Anat. 4: $338-364$.

Huziwara, Y. 1962. Karyotype analysis in some genera of Compositae VIII. Further studies on the chromosome of Aster. Amer. J. Bot. 49: 116-119.

Krishnaswamy, N, and Ayyanger, Rangaswamy, G. N. 1935. Chromosome numbers in Cajanus cajan Spreng. Curr. Sci. 3: 614-615.

Nathaini, S. P. 1941. Cytological studies on Indian pulses. Part I. Proc. Nat. Acad. Sci. India. 11: $67-73$.

Sato, D. 1937. Karyotype alteration and phylogeny. Cytologia Fugii Jubilee Volume. : 80-95.

Shaw, F. J. F., Khan, A. R. and Singh, N. 1933. Studies in Indian pulses. The type of Cajanus indicus Spreng, Indian J. Agric. Sci. 3(1): 1-36.

Sinha, S. S. N. and Acharia, S. S. 1972. Karyotype analysis in some varieties of Lens culinaris. Cytologia 37: 673-683.

Shrivastava, M. P., Sharma, D. and Singh, Laxman 1973. Karyotype analysis on fifteen varieties of C. cajan L. (Mill sp.) and Autylosia lineata (W. and A.), Cytologia 38(2): 219-227.

Stebbins, G. L. Jr. 1950. Variation and Evolution in Plants. Columbia Univ. Press. New York. 